\begin{tabular}{|c|c|}
\hline Title & Coupling motion between rearfoot and hip and knee joints during walking and single leg landing \\
\hline Author(s) & $\begin{array}{l}\text { Koshino, Y uta; Y amanaka, Masanori; Ezawa, Y uya; Okunuki, Takumi; Ishida, Tomoya; Samukawa, Mina; Tohyama, } \\
\text { Harukazu }\end{array}$ \\
\hline Citation & $\begin{array}{l}\text { Journal of electromy ography and kinesiology, 37, 75-83 } \\
\text { https://doi.org/10.1016/.j.jelekin.2017.09.004 }\end{array}$ \\
\hline Issue Date & $2017-12$ \\
\hline Doc URL & http:/hdl .handle.net/2115/72264 \\
\hline Rights & $\begin{array}{l}\text { () 2017. This manuscript version is made available under the CC-BY-NC-ND } 4.0 \text { license } \\
\mathrm{http}: / / \text { reativecommons.org/icenses/by-nc-nd/4.0/ }\end{array}$ \\
\hline Rights(URL) & http://creativecommons.org/icenses/by-nc-nd/4.0/ \\
\hline Type & article (author version) \\
\hline File Information & J Electromyogr Kinesiol_37_75-83.pdf \\
\hline
\end{tabular}

Instructions for use 


\section{Coupling motion between rearfoot and hip and knee joints during walking and single-leg landing}

Yuta Koshino $^{1,2}$, Masanori Yamanaka ${ }^{1}$ Yuya Ezawa $^{3}$, Takumi Okunuki ${ }^{3}$, Tomoya Ishida ${ }^{1,4}$, Mina Samukawa ${ }^{1}$, Harukazu Tohyama ${ }^{1}$

1. Faculty of Health Sciences, Hokkaido University, Sapporo, Hokkaido, Japan.

2. Rehabilitation Center, NTT East Japan Sapporo Hospital, Sapporo, Hokkaido, Japan.

3. Department of Rehabilitation, Matsuda Orthopedic Memorial Hospital, Sapporo, Hokkaido, Japan.

4. Department of Rehabilitation, Hokushin Orthopedic Hospital, Sapporo, Hokkaido, Japan.

Key Words: kinetic chain; foot pronation; ankle kinematics; lower limb; cross correlation; hindfoot; vector coding technique

Corresponding author: Masanori Yamanaka, PT, Ph.D.

Faculty of Health Science, Hokkaido University

West-5, North-12, Kita-ku, Sapporo, 060-0812, Japan.

Tell: +81-11-706-3383, Fax: +81-11-706-3383

E-mail: yamanaka@hs.hokudai.ac.jp 


\section{Abstract}

2 The objective of the current study was to investigate the kinematic relationships between the

3 rearfoot and hip/knee joint during walking and single-leg landing. Kinematics of the rearfoot

4 relative to the shank, knee and hip joints during walking and single-leg landing were analyzed

$5 \quad$ in 22 healthy university students. Kinematic relationships between two types of angular data

6 were assessed by zero-lag cross-correlation coefficients and coupling angles, and were

7 compared between joints and between tasks. During walking, rearfoot eversion/inversion and

8 external/internal rotation were strongly correlated with hip adduction/abduction $(R=0.69$ and

$9 \quad R=0.84)$, whereas correlations with knee kinematics were not strong $(R \leq 0.51)$ and varied

10 between subjects. The correlations with hip adduction/abduction were stronger than those

11 with knee kinematics $(P<0.001)$. Most coefficients during single-leg landing were strong $(R$

$12 \geq 0.70)$, and greater than those during walking $(P<0.001)$. Coupling angles indicated that hip

13 motion relative to rearfoot motion was greater than knee motion relative to rearfoot motion

14 during both tasks $(P<0.001)$. Interventions to control rearfoot kinematics may affect hip

15 kinematics during dynamic tasks. The coupling motion between the rearfoot and hip/knee

16 joints, especially in the knee, should be considered individually. 


\section{Introduction}

The kinematics of the foot and ankle affect proximal joints kinematics, such as hip and knee joints, during both static and dynamic conditions (Khamis and Yizhar, 2007; Resende et al., 2015; Tateuchi et al., 2011). This linkage between foot/ankle and the proximal joints may contribute to musculoskeletal injuries in the lower limbs (Chuter et al., 2012). For example, the pathology of patellofemoral pain syndrome (Barton et al., 2009) and medial tibial stress syndrome (Viitasalo et al., 1983) are reported to be related to dynamic foot function. In addition, knee valgus, which is a risk factor for anterior cruciate ligament injury, has been partially attributed to excessive foot pronation (Joseph et al., 2008). Excessive foot and ankle motion may be associated with a variety of sports injuries in the lower limbs.

The effects of foot and ankle kinematics on lower limb joint kinematics have been investigated in a small number of studies. Induced hyperpronation of the foot by wedges was found to result in increases in internal rotation of both the knee joint and the hip joint during standing (Khamis and Yizhar, 2007), increased hip internal rotation during single-leg standing (Tateuchi et al., 2011), and increased internal rotation of the hip joint, femur and shank, as well as changes in the temporal pattern of knee internal rotation during walking (Resende et al., 2015). However, these studies examined the effect of the hyperpronation of the foot induced by wedges, which may be beyond the range of normal foot motion. In a previous study that did not induce foot motion, rearfoot eversion was found to be synchronized with hip internal rotation (Souza et al., 2010) and correlated with hip adduction and shank internal rotation during the stance phase of walking (Barton et al., 2012). However, to our knowledge, the effects of rearfoot kinematics on the kinematics of the hip and knee joints have only been examined during walking, and have not been examined during sports-related tasks such as jump-landing, which is involved in a variety of sports and is associated with musculoskeletal injuries of the lower limbs (Doherty et al., 2016; van der Does et al. 2016). Thus, examining 
coupling motion during a landing task could provide basic information for assessment of joint kinematics and for prevention and rehabilitation of musculoskeletal injuries of the lower limbs in clinical settings.

Lafortune et al. (1994) examined the effects of a $10^{\circ}$ pronation wedge and a $10^{\circ}$ supination wedge on knee kinematics using bone-pins during walking. Their results revealed only minor changes in the knee angular pattern, suggesting that foot kinematics had a weak effect on knee joint kinematics during walking. Although the findings suggested that tibial rotation induced by pronation/supination wedges was resolved at the hip joint rather than the knee joint, hip joint kinematics were not measured in the previous study (Lafortune et al., 1994). Importantly, it is currently unclear whether foot kinematics have a stronger association with the hip or knee joint during dynamic tasks. It is important for clinicians to understand the interrelationships within the lower limb kinematics to address malposition of the hip or knee joint. The current study had three main aims: to investigate the kinematic relationships between the rearfoot and the hip and knee joints during walking and single-leg landing, to investigate whether the relationship between the rearfoot and hip joint differed from those between the rearfoot and knee joint, and to compare those relationships between walking and single-leg landing. We hypothesized that rearfoot kinematics would be associated with hip joint kinematics, whereas rearfoot kinematics would not be associated with knee joint kinematics during both tasks.

\section{Methods}

\subsection{Subjects}

Twenty-two healthy university students participated in this study (11 males, 11 females, age: 21.9 (1.1) years old, height: 167.2 (8.4) cm, body weight: 57.4 (6.6) kg). A priori power analysis in $\mathrm{G}^{*}$ Power 3.1.7 was performed using the correlation coefficients 
between rearfoot and hip joint motion in a previous study (Souza et al., 2010). As a result, at least 22 subjects were required to achieve statistical power of $80 \%$ with an alpha level of 0.05 for the correlation analyses. All participants had no history of surgery or fracture in the lower limbs, and had no musculoskeletal injuries within the past 6 months. Because the dominant side (the side used for kicking a ball) was the right leg in all subjects, the right lower limbs were tested and analyzed. The experiments were performed after gaining ethical committee approval from the University Institutional Review Board. Informed consent was obtained from all subjects.

\subsection{Procedure}

Six high-speed digital cameras (Hawk cameras, Motion Analysis Corporation, Santa Rosa, CA, USA) and a force plate (Type 9286, Kistler AG, Winterthur, Switzerland) were time-synchronized and used for motion analysis during walking and single-leg landing. Reflective markers were attached to the bilateral anterior superior iliac spine, sacral, lateral thigh, and lateral and medial femoral epicondyles. Markers of the shank and foot were attached to the tibial tuberosity, the head of the fibula, lateral and medial malleoli, Achilles’ tendon attachment, posterior surface of the calcaneus, peroneal tubercle, sustentaculum tali, tuberosity of the navicular, base of the first, second and fifth metatarsal, head of the first, second and fifth metatarsal, and head of the proximal phalanx of the hallux, based on the Rizzoli multi-segment foot model (Figure 1) (Leardini et al., 2007). EvaRT 4.3.57 (Motion Analysis Corporation) software was used to record the marker coordinates during each task, sampled at $200 \mathrm{~Hz}$ for kinematic data and $1000 \mathrm{~Hz}$ for force data.

For the walking task, subjects walked at their natural speed. For single-leg landing, subjects dropped from a 30-cm box from their left leg, and landed with the right leg on the force plate. Subjects practiced up to 10 trials of each task before recording, and performed 
three successful trials for each task. Trials in which the entire right foot landing on the force plate, the left foot did not touch the force plate, and the subject did not lose balance during testing were defined as successful trials.

\subsection{Data collection and reduction}

Kinematic data were low-pass filtered using a $4^{\text {th }}$ order Butterworth filter with a $6 \mathrm{~Hz}$ cutoff frequency. Hip and knee joint angles were calculated using the traditional lower limb model (Helen Hayes model), and the rearfoot angle was calculated using the Rizzoli multi-segment foot model (Leardini et al., 2007) using Visual 3D software (C-Motion Inc., Germantown, MD, USA). The Rizzoli multi-segment foot model has five segments, as follows: shank, rearfoot, midfoot, forefoot and hallux. In the current study, the rearfoot angle with respect to the shank was calculated according to the joint coordinate system (Grood and Suntay, 1983). The angle data were extracted from initial contact to toe off during walking and from initial contact to maximum knee flexion during single-leg landing. The initial contacts during the both tasks were defined as the time at which the vertical ground reaction force first exceeded $10 \mathrm{~N}$, while toe-off during walking was defined as the time at which force first fell below $10 \mathrm{~N}$ after initial contact. Joint and segment angles were set to zero during a static standing position with the hip joints in a neutral position in the frontal plane and the toes facing straight forward. The adduction and internal rotation of the hip and knee joints were represented as positive values, while eversion and external rotation of rearfoot relative to shank were represented as positive values.

Cross-correlation analysis was used to assess kinematic coupling between the hip and knee joints and the rearfoot during each task. Cross-correlation analysis can determine the strength of the temporal relationship between two time-series angular data sets (Souza et al., 2010; Pohl et al., 2007). A cross-correlation coefficient is high when the two curves of angular 
data sets have similar timing and shape (Wren et al., 2006). Zero-lag normalized cross correlation was calculated in the current study, because this represents the strength of the relationship between two time-series angular data sets in real time, and the coefficient with time lags between rearfoot and hip joint angular data was no stronger than that with a zero time lag (Souza et al., 2010). Zero-lag normalized cross correlation analysis was performed based on an equation described in a previous report (Nelson-Wong et al., 2009) using Matlab (The MathWorks Inc, Natick, MA):

$$
R_{x y}=\frac{\frac{1}{N} \sum_{n=1}^{N}\left(x_{n}-\bar{x}\right)\left(y_{n}-\bar{y}\right)}{\frac{1}{N} \sqrt{\sum_{n=1}^{N}\left(x_{n}-\bar{x}\right)^{2} \sum_{n=1}^{N}\left(y_{n}-\bar{y}\right)^{2}}}
$$

where $R_{x y}$ is the correlation coefficient of two time-series data $x$ and $y$ and $N$ represent the number of data points. The coefficients between the following angular data during walking and single-leg landing were calculated for each motion trial of each subject: hip adduction (ADD)/abduction (ABD) and rearfoot eversion (EVE)/inversion (INV), hip ADD/ABD and rearfoot external rotation (ER)/internal rotation (ER), hip IR/ER and rearfoot EVE/INV, hip $\mathrm{IR} / \mathrm{ER}$ and rearfoot ER/IR, knee $\mathrm{ADD} / \mathrm{ABD}$ and rearfoot EVE/INV, knee ADD/ABD and rearfoot ER/IR, knee IR/ER and rearfoot EVE/INV, and knee IR/ER and rearfoot ER/IR (Table 1). Interpretations of the coefficients were as follows: very strong ( 0.80 to 1.00 or -0.80 to -1.00$)$, strong ( 0.60 to 0.79 or -0.60 to -0.79$)$, moderate $(0.40$ to 0.59 or -0.40 to $-0.59)$, weak ( 0.20 to 0.39 or -0.20 to -0.39 ) and very weak ( 0 to 0.19 or 0 to -0.19 ) (Campbell and Swinscow, 2009).

Since the results of zero-lag normalized cross correlation analysis were not reflected by the magnitude of the time-series angular data, the analysis cannot assess the quantity of coupling motions. Therefore, a vector coding (coupling angle) technique was used to assess the quantity of kinematic couplings of interest, based on an equation described in a previous report (Pohl et al., 2007): 


$$
\Theta_{i}=\operatorname{abs}\left[\tan ^{-1}\left(\frac{y_{i+1}-y_{i}}{x_{i+1}-x_{i}}\right)\right]
$$

where $\Theta_{i}$ is the coupling angle between proximal $(x)$ and distal $(y)$ joint angles, and $i$ represents the number of data points. The proximal joint motion is greater when the coupling angle $<45^{\circ}$, whereas the distal joint motion is greater when the coupling angle $>45^{\circ}$. The mean coupling angles were calculated for the period of interest.

\subsection{Statistical analysis}

Zero-lag normalized cross correlation coefficients and coupling angles of each subject were set as the dependent variables. Shapiro-Wilk tests revealed that most coefficients were not normally distributed, while all coupling angles other than that of rearfoot EVE/INV and knee IR/ER were normally distributed. Thus, Wilcoxon signed-rank tests and paired t-tests were used to investigate whether the strength of correlations and coupling angles between the rearfoot and hip joint differed from the strength of correlations between the rearfoot and the knee joint, respectively. The significance level was set at 0.0125 with a Bonferroni correction based on the four comparisons: 1) rearfoot EVE/INV or ER/IR and hip $\mathrm{ADD} / \mathrm{ABD}$ vs. rearfoot EVE/INV or ER/IR and knee ADD/ABD; 2) rearfoot EVE/INV or ER/IR and hip ADD/ABD vs. rearfoot EVE/INV or ER/IR and knee IR/ER; 3) rearfoot EVE/INV or ER/IR and hip IR/ER vs. rearfoot EVE/INV or ER/IR and knee ADD/ABD; 4) rearfoot EVE/INV or ER/IR and hip IR/ER vs. rearfoot EVE/INV or ER/IR and knee IR/ER (Table 1). In addition, Wilcoxon signed-rank tests and paired t-tests were used to compare the coefficients and coupling angles between walking and single-leg landing, respectively, and the significance level was set at 0.0125 with a Bonferroni correction based on the four comparisons of rearfoot EVE/INV and rearfoot ER/IR.

\section{Results}




\subsection{Walking}

Averaged time-series angular displacements of hip joint, knee joint, and rearfoot relative to the shank during walking are shown in Figure 2. Rearfoot EVE/INV was strongly correlated with hip ADD/ABD (median $R=0.69$ ), and the correlation was significantly stronger than the correlations between the rearfoot EVE/INV and knee ADD/ABD and that between the rearfoot EVE/INV and knee IR/ER (all: $P<0.001$; Table 2). Most subjects exhibited greater than or equal to moderate correlations between rearfoot EVE/INV and hip ADD/ABD (Figure 3). Rearfoot EVE/INV had weak correlations with hip IR/ER, knee ADD/ABD and knee IR/ER (median $R=0.06,0.37$ and -0.04 , respectively) (Table 2). These correlations varied considerably between subjects (Figure 3). Rearfoot ER/IR had a very strong correlation with hip ADD/ABD (median $R=0.84$ ), and the correlation was significantly stronger than the correlation between rearfoot ER/IR and knee ADD/ABD, and that between rearfoot ER/IR and knee IR/ER (all: $P<0.001$; Table 2). Most subjects exhibited greater than or equal to moderate correlations between rearfoot ER/IR and hip ADD/ABD (Figure 3). Rearfoot ER/IR had weak to moderate correlations with hip IR/ER, knee $\mathrm{ADD} / \mathrm{ABD}$ and knee IR/ER (median $R=-0.26, R=0.51$ and -0.41 , respectively) (Table 2), and these correlations varied considerably between subjects (Figure 3).

The coupling angles between the rearfoot and hip joint were significantly smaller than those between the rearfoot and knee joint during walking (all: $P<0.001$; Table 3). This result indicated that hip joint motion relative to rearfoot motion was greater than knee joint motion relative to rearfoot motion. The mean coupling angles indicated slightly greater hip $\mathrm{ADD} / \mathrm{ABD}$ and IR/ER motion relative to rearfoot motion, and less knee ADD/ABD motion and slightly less knee IR/ER motion relative to rearfoot motion (Table 3).

\subsection{Single-leg landing}


Averaged time-series angular displacements of the hip joint, knee joint, and rearfoot relative to shank during single-leg landing are shown in Figure 4. Rearfoot EVE/INV had a very strong correlation with hip IR/ER and knee IR/ER (median $R=0.89$ and 0.87, respectively) and a strong correlation with hip $\mathrm{ADD} / \mathrm{ABD}$ and knee $\mathrm{ADD} / \mathrm{ABD}$ (median $R=$ 0.70 and 0.79 , respectively) (Table 4). However, some subjects exhibited a weak or negative correlation (Figure 5). The correlations with rearfoot EVE/INV were not significantly different between the hip and knee. Rearfoot ER/IR had a very strong correlation with hip ADD/ABD, hip IR/ER and knee ADD/ABD (median $R=0.92,0.92$ and 0.80, respectively), and was strongly correlated with knee IR/ER (median $R=0.79$ ) (Table 4). Although most subjects exhibited greater than or equal to strong correlations between rearfoot ER/IR and hip ADD/ABD or IR/ER, some subjects exhibited a weak or negative correlation between rearfoot ER/IR and knee ADD/ABD or IR/ER (Figure 5). The correlation between rearfoot ER/IR and hip ADD/ABD was significantly stronger than the correlation between rearfoot ER/IR and knee IR/ER $(P=0.001)$ and was no different to the other correlations (Table 4). The coupling angles between the rearfoot and hip joint were significantly less than those between the rearfoot and knee joint (all: $P<0.001$; Table 5). Therefore, hip joint motion relative to rearfoot motion was greater than knee joint motion relative to rearfoot motion. The mean coupling angles indicated slightly greater hip joint motion and slightly less knee joint motion, relative to rearfoot motion (Table 5).

\subsection{Comparison between the tasks}

A comparison between the walking and single-leg landing conditions revealed that the correlations between rearfoot EVE/INV and hip IR/ER, and those between rearfoot EVE/INV and knee IR/ER were significantly stronger in the single-leg landing condition than in the walking condition (both, $P<0.001$ ). The correlations between rearfoot ER/IR and hip 
$\mathrm{ADD} / \mathrm{ABD}$, between rearfoot ER/IR and hip IR/ER, and between rearfoot ER/IR and knee IR/ER were also significantly stronger in the single-leg landing condition compared with the walking condition (all, $P<0.001$ ).

The coupling angles between rearfoot EVE/INV and hip IR/ER, between rearfoot EVE/INV and knee ADD/ABD, and between rearfoot ER/IR and knee ADD/ABD were significantly smaller in the single-leg landing condition compared with the walking condition ( $P=0.001, P<0.001$, and $P<0.001$, respectively). This indicates that hip IR/ER and knee $\mathrm{ADD} / \mathrm{ABD}$ motion relative to rearfoot motion was greater in the single-leg landing than in the walking condition.

\section{Discussion}

The current study revealed that rearfoot motion in the frontal and horizontal planes was most strongly correlated with hip frontal plane motion during walking. However, other correlations were not strong, and varied considerably between individuals during walking. In the single-leg landing condition, all correlations between rearfoot and hip and knee joints ranged from strong to very strong, and most correlations were significantly stronger than those in the walking condition. In addition, although proximal joint motion relative to rearfoot motion was greater in the hip joint than in the knee joint during both tasks, some of the relative proximal joint motions were greater in single-leg landing than in walking. These findings suggest that the strength of kinematic relationships and the relative amount of coupling motion with rearfoot motion differed between the hip and knee joints, and was task-dependent.

Rearfoot EVE/INV and ER/IR motion were the most strongly correlated with hip ADD/ABD motion during walking. Rearfoot EVE and ER would be expected to cause internal rotation of the shank and femur under closed-kinetic conditions (Khamis and Yizhar, 
2007; Resende et al., 2015). Since the knee position is slightly flexed during most of the stance phase of walking (Lafortune et al., 1992), shank and femur internal rotation may shift the knee medially, resulting in hip adduction. The present findings are partially in accord with those of a previous study by Barton et al. (2012), who reported that the range of motion in rearfoot eversion was associated with the peak value and range of motion in hip adduction kinematics during walking in healthy subjects and patellofemoral pain syndrome subjects. However, this previous study assessed the kinematic relationship with Pearson's correlation coefficients using discrete variables, and did not assess the relationship with temporal kinematic patterns (Barton et al, 2012). In the present study, the kinematic patterns of the rearfoot were synchronized with the pattern of hip ADD/ABD motion. The amount of hip $\mathrm{ADD} / \mathrm{ABD}$ motion was slightly greater relative to that of rearfoot motion in the coupling motion, as indicated by the coupling angle. The present findings indicated that interventions to control the motion pattern of rearfoot eversion and external rotation may affect hip adduction motion patterns during walking, and vice versa.

The temporal kinematic relationships between the rearfoot and knee joint were found to be relatively weak and varied considerably between subjects during walking in the present study. The relative amount of coupling motion with the rearfoot was also less in the knee joint than the hip joint. Lafortune et al. (1994) reported that foot inversion or eversion induced by $10^{\circ}$ valgus or varus wedges caused minor changes in knee joint motion (less than $1^{\circ}$ ) during walking. Foot pronation induced by $10^{\circ}$ wedged sandal resulted in constant increase in the hip internal rotation, but no constant increase in the knee internal rotation during walking (Resende et al., 2015). In another study, foot posture (such as the planus and cavus foot types) also had only minor effects on knee joint motion and moment during walking (Buldt et al., 2015). The ligament and muscles at the knee joint may resist the kinematic chain through shank motion induced by rearfoot motion during walking (Souza et al.. 2010; Lafortune et al., 
1994). Therefore, foot motion may not strongly affect knee motion during walking. However, the kinematic relationships should be considered individually, because some subjects exhibited a strong relationship (Figure 3).

In the current study, in the single-leg landing condition, rearfoot EVE/INV and ER/IR motion had very strong or strong correlations with hip joint motion, as well as knee joint motion. In addition, hip IR/ER and knee ADD/ABD motion relative to rearfoot motion were greater in single-leg landing than in walking. A previous study found that changing foot position during drop vertical jump significantly affected knee kinematics and kinetics in the frontal and horizontal planes (Ishida et al., 2015). Rearfoot motion may have major effects on knee and hip joint motion during the landing task. Single-leg landing may have resulted in decreased compliance of the soft tissues via strong contraction of the muscles around the ankle to attenuate landing impact (Yeow et al., 2011). Stiffness around the joint may result in a strong correlation between the rearfoot and hip and knee kinematics, as Pohl et al. (2007) speculated. In addition, the joint angular velocities in most joints of the lower limbs would be expected to be high during single-leg landing (Dowling et al., 2012). Subjects may have had difficulty controlling rapid joint motion with the muscles, meaning that rearfoot motion may have been predisposed to link both the knee and hip joint during single-leg landing. The results of the current study revealed considerable individual variation in some coupling motions. A previous study investigating coupling motion between rearfoot EVE/INV and hip IR/ER in the walking stance suggested that coupling strength should be considered individually in clinical settings (Souza et al., 2010). The current study also suggests that the coupling motion between rearfoot and hip IR/ER during walking should be considered individually. Furthermore, the current findings suggest that the coupling motion between the rearfoot and knee joint should also be considered individually during not only walking but also single-leg landing. The variation in the coupling motion may be caused by multiple 
factors, such as foot posture, lower limb alignment, joint laxity and muscle function. Future studies are required to clarify the cause of this individual variation.

The present study involved several limitations that should be considered. First, the coupling motion between the rearfoot and hip and knee joints in other tasks may differ from the present findings because the characteristics of the coupling motion were task-dependent. Future studies should investigate coupling motion during running, cutting maneuvers or double-leg landing tasks. Second, we used cross-correlation analysis between two types of angular data based on the assumption that they would be independent. In actuality, two angular data types would not be expected to be completely independent because of mechanical connections between joints. The coupling motion types observed in the present study would include the mechanical coupling and conscious coupling. Finally, the present study examined only healthy subjects. Subjects with musculoskeletal injury or surgery in the lower limbs may exhibit different coupling motion patterns from the current findings.

\section{Conclusion}

The current study found that rearfoot EVE/INV and ER/IR motion were most strongly correlated with hip ADD/ABD motion, while the correlations between the rearfoot motion and knee joint motion were not strong and varied between individuals during walking. In the single-leg landing condition, rearfoot EVE/INV and ER/IR motion were strongly correlated with both hip and knee joint motion. The hip joint motion relative to rearfoot motion was greater than the knee joint motion relative to rearfoot motion in the coupling motion during both tasks. These findings suggest that the strength of kinematic relationships and the relative amount of coupling motion with rearfoot motion differed between the hip and knee joints, and that this effect was task-dependent. 


\section{Acknowledgements}

We thank Benjamin Knight, MSc, from Edanz Group (www.edanzediting.com/ac) for editing a draft of this manuscript.

\section{References}

Barton CJ, Levinger P, Crossley KM, Webster KE, Menz HB. The relationship between rearfoot, tibial and hip kinematics in individuals with patellofemoral pain syndrome. Clin Biomech. 2012;27(7):702-705.

Barton CJ, Levinger P, Menz HB, Webster KE. Kinematic gait characteristics associated with patellofemoral pain syndrome: a systematic review. Gait Posture. 2009;30(4):405-416.

Buldt AK, Levinger P, Murley GS, Menz HB, Nester CJ, Landorf KB. Foot posture and function have only minor effects on knee function during barefoot walking in healthy individuals. Clin Biomech. 2015;30(5):431-437.

Campbell MJ, Swinscow TDV. Statistics at Square One. 11th ed. Chichester: Wiley-Blackwell, 2009.

Chuter VH, Janse de Jonge XAK. Proximal and distal contributions to lower extremity injury: a review of the literature. Gait Posture. 2012;36(1):7-15.

Doherty C, Bleakley C, Hertel J, Caulfield B, Ryan J, Delahunt E. Single-leg drop landing movement strategies in participants with chronic ankle instability compared with lateral ankle sprain ‘copers’. Knee Surg Sports Traumatol Arthrosc. 2016;24(4):1049-1059.

Dowling AV, Favre J, Andriacchi TP. Characterization of thigh and shank segment angular velocity during jump landing tasks commonly used to evaluate risk for ACL injury. $\mathrm{J}$ Biomech Eng. 2012;134(9):091006.

Grood ES, Suntay WJ. A joint coordinate system for the clinical description of three-dimensional motions: application to the knee. J Biomech Eng. 1983;105(2):136-44. 
Ishida T, Yamanaka M, Takeda N, et al., The effect of changing toe direction on knee kinematics during drop vertical jump: a possible risk factor for anterior cruciate ligament injury. Knee Surg Sports Traumatol Arthrosc. 2015;23(4):1004-1009.

Joseph M, Tiberio D, Baird JL, et al. Knee valgus during drop jumps in National Collegiate Athletic Association Division I female athletes: the effect of a medial post. Am J Sports Med. 2008;36(2):285-289.

Khamis S, Yizhar Z. Effect of feet hyperpronation on pelvic alignment in a standing position. Gait Posture. 2007;25(1):127-134.

Lafortune MA, Cavanagh PR, Sommer HJ, Kalenak A. Foot inversion-eversion and knee kinematics during walking. J Orthop Res. 1994;12(3):412-420.

Lafortune MA, Cavanagh PR, Sommer HJ, Kalenak A. Three-dimensional kinematics of the human knee during walking. J Biomech. 1992;25(4):347-357.

Leardini A, Benedetti MG, Berti L, Bettinelli D, Nativo R, Giannini S. Rear-foot, mid-foot and fore-foot motion during the stance phase of gait. Gait Posture. 2007;25(3):453-462.

Nelson-Wong E, Howarth S, Winter DA, Callaghan JP. Application of autocorrelation and cross-correlation analyses in human movement and rehabilitation research. J Orthop Sports Phys Ther. 2009;39(4):287-295.

Pohl MB, Messenger N, Buckley JG. Forefoot, rearfoot and shank coupling: effect of variations in speed and mode of gait. Gait Posture. 2007;25(2):295-302.

Resende RA, Deluzio KJ, Kirkwood RN, Hassan EA, Fonseca ST. Increased unilateral foot pronation affects lower limbs and pelvic biomechanics during walking. Gait Posture. 2015;41(2):395-401.

Souza TR, Pinto RZ, Trede RG, Kirkwood RN, Fonseca ST. Temporal couplings between rearfoot-shank complex and hip joint during walking. Clin Biomech. 2010;25(7):745-748. 
349 Tateuchi H, Wada O, Ichihashi N. Effects of calcaneal eversion on three-dimensional

350 kinematics of the hip, pelvis and thorax in unilateral weight bearing. Hum Mov Sci.

$351 \quad 2011 ; 30(3): 566-573$.

352 Van der Does HTD, Brink MS, Benjaminse A, Visscher C, Lemmink KAPM. Jump landing

353 characteristics predict lower extremity injuries in indoor team sports. Int J Sports Med.

$354 \quad 2016 ; 37(3): 251-256$.

355 Viitasalo JT, Kvist M. Some biomechanical aspects of the foot and ankle in athletes with and 356 without shin splints. Am J Sports Med. 1983;11(3):125-130.

357 Wren TAL, Do KP, Rethlefsen SA, Healy B. Cross-correlation as a method for comparing 358 dynamic electromyography signals during gait. J Biomech. 2006;39(14):2714-2718.

359 Yeow CH, Lee PV, Goh JC. An investigation of lower extremity energy dissipation strategies 360 during single-leg and double-leg landing based on sagittal and frontal plane biomechanics. 361 Hum Mov Sci. 2011;30(3):624-635. 


\section{Figure captions}

Figure 1. Marker location.

Figure 2. Averaged time-series angle data (mean $\pm \mathrm{SD}$ ) during walking. The horizontal axis indicates the stance phase from initial contact (0\%) to toe-off (100\%).

Figure 3. Frequency diagram of the correlation coefficients between rearfoot motion and knee and hip motion during walking in 22 subjects. The vertical axis indicates the number of subjects. The horizontal axis indicates the correlation coefficient.

Figure 4. Averaged time-series angle data (mean \pm SD) during single-leg landing. The horizontal axis indicates the landing phase from initial contact (0\%) to maximum knee flexion (100\%).

Figure 5. Frequency diagram of the correlation coefficients between rearfoot motion and knee and hip motion during single-leg landing in 22 subjects. The vertical axis indicates the number of subjects. The horizontal axis indicates the correlation coefficient. 
$\bullet$

$x$

3
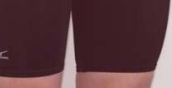

b

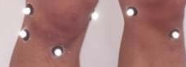


Hip ADD(+)/ABD(-)
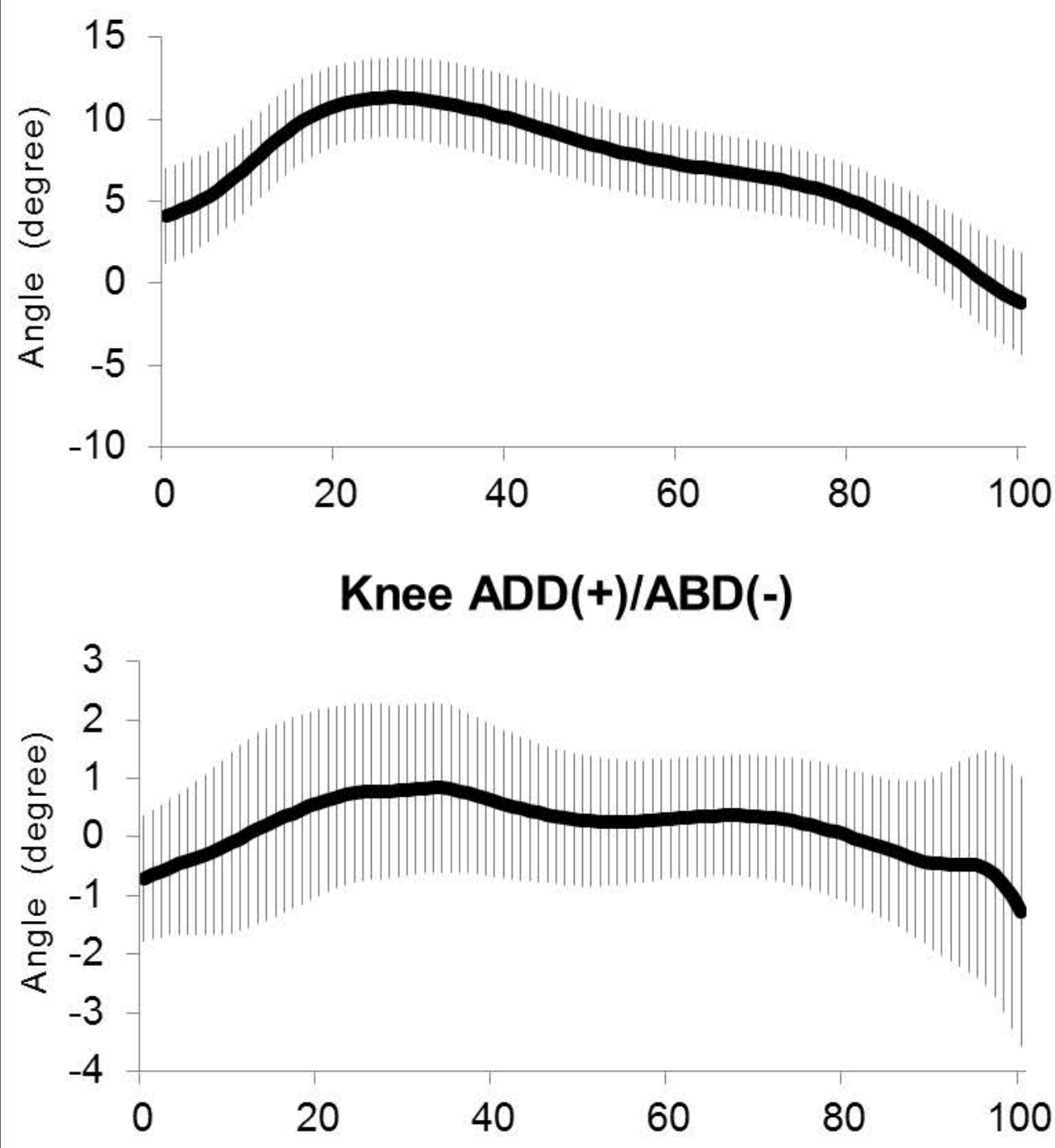

Rearfoot EVE(+)/INV(-)

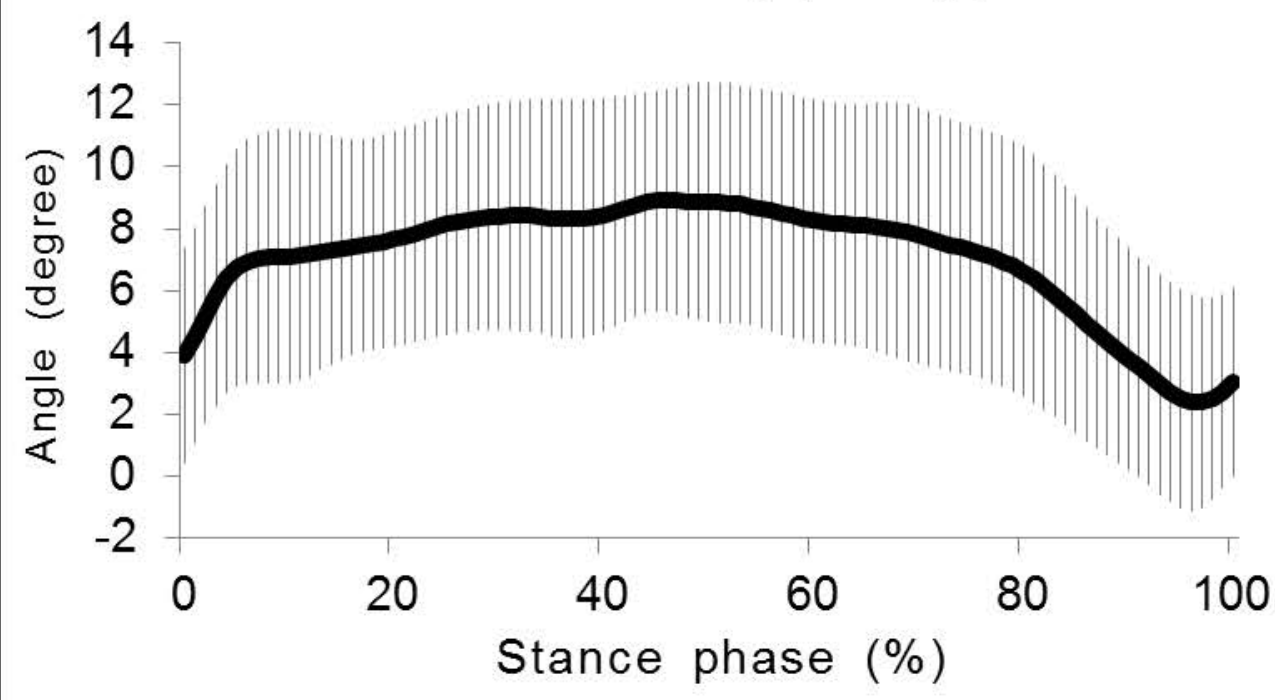

Hip IR(+)/ER(-)

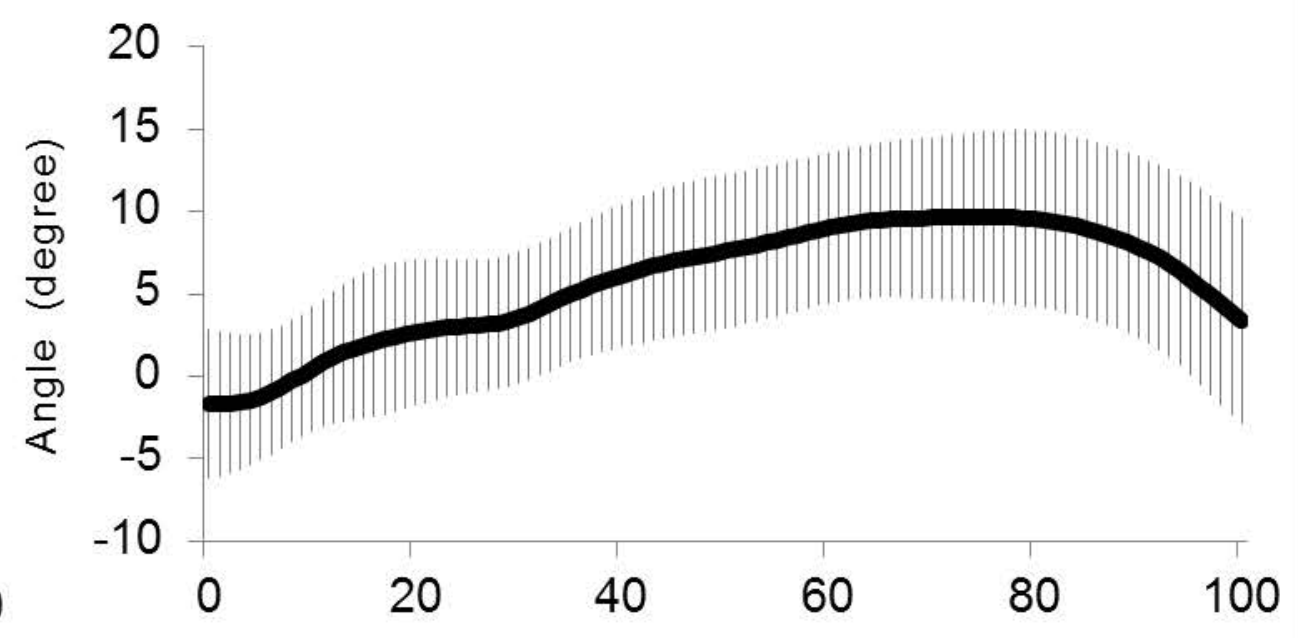

Knee IR(+)/ER(-)

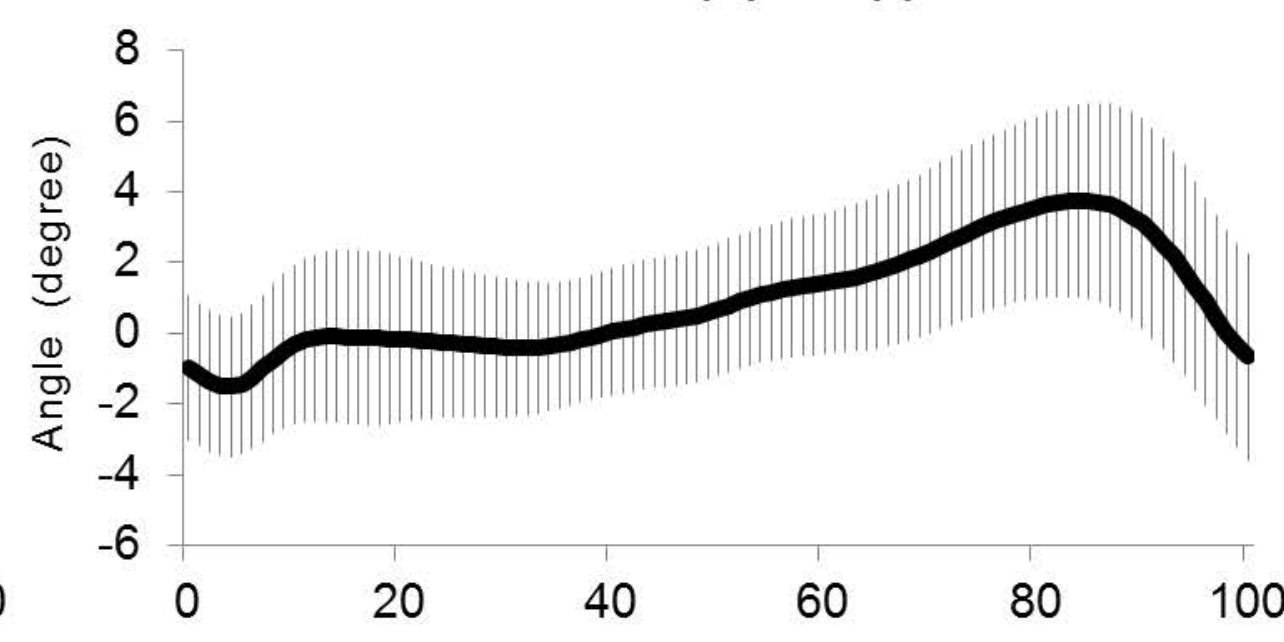

Rearfoot ER(+)/IR(-)

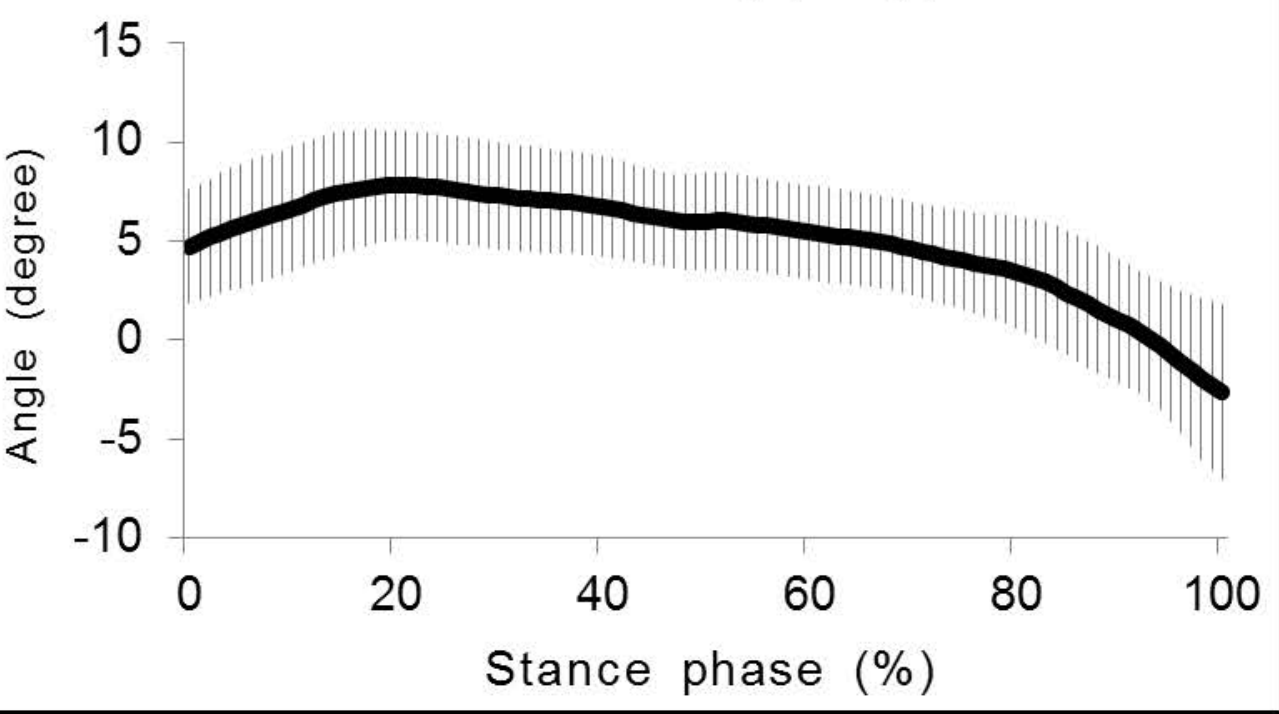


Correlation between

rearfoot EVE/INV and hip ADD/ABD

20

0

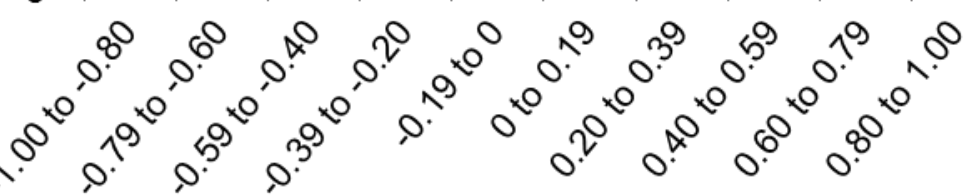

Correlation between

rearfoot EVE/INV and knee ADD/ABD

20
15
10
5
0

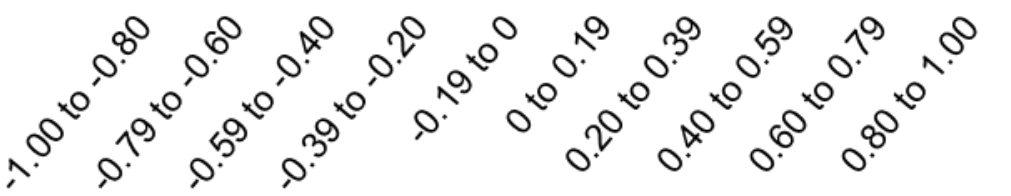

Correlation between

rearfoot ER/IR and hip ADD/ABD

20
15
10
5
0

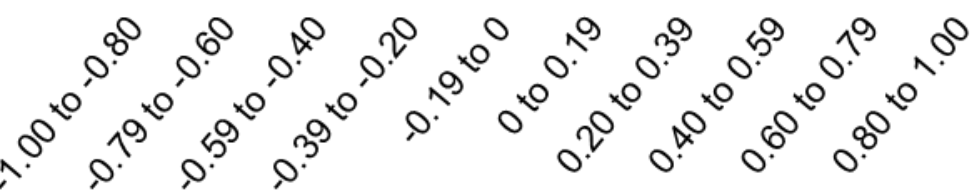

Correlation between

rearfoot ER/IR and knee ADD/ABD

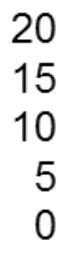

20
15
10
5
0

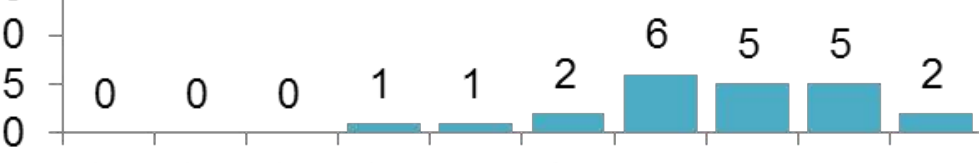

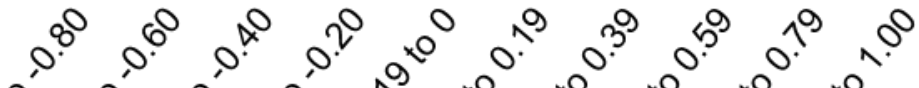

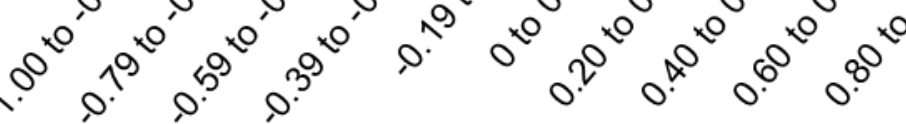

Correlation between rearfoot EVE/INV and hip IR/ER

10

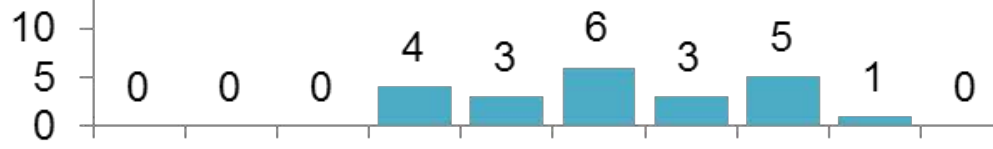

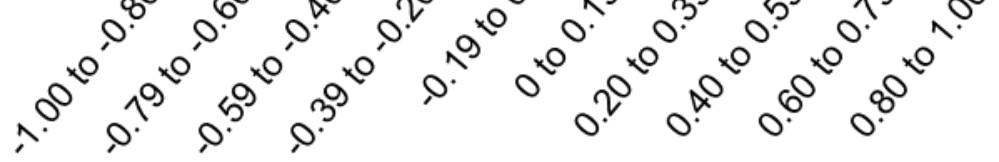

Correlation between rearfoot EVE/INV and knee IR/ER

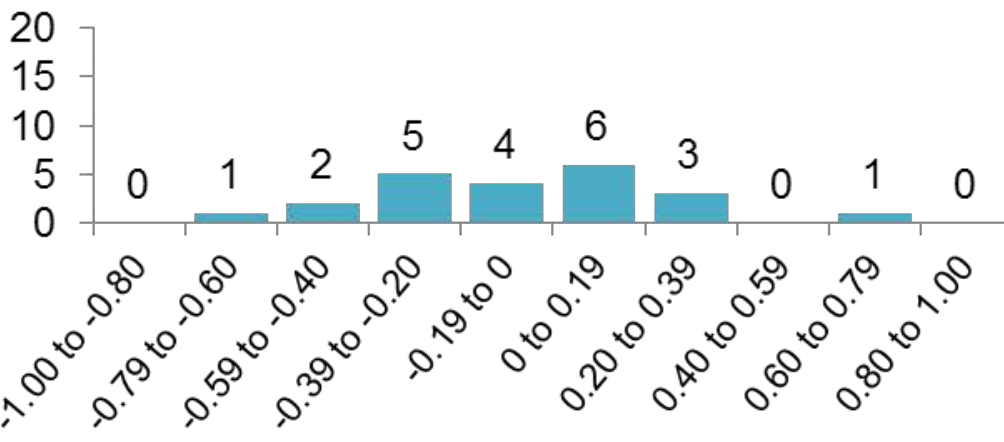

Correlation between rearfoot ER/IR and hip IR/ER

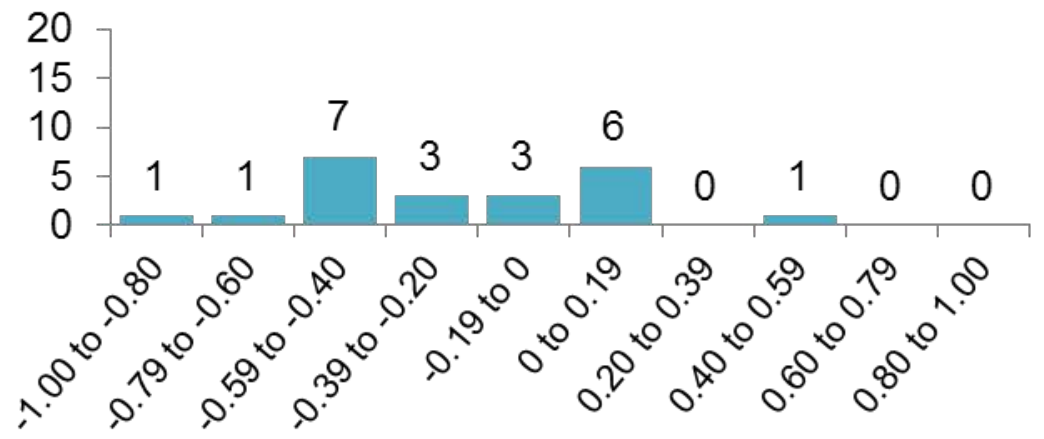

Correlation between rearfoot $E R / I R$ and knee IR/ER

20

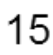

10

5
0
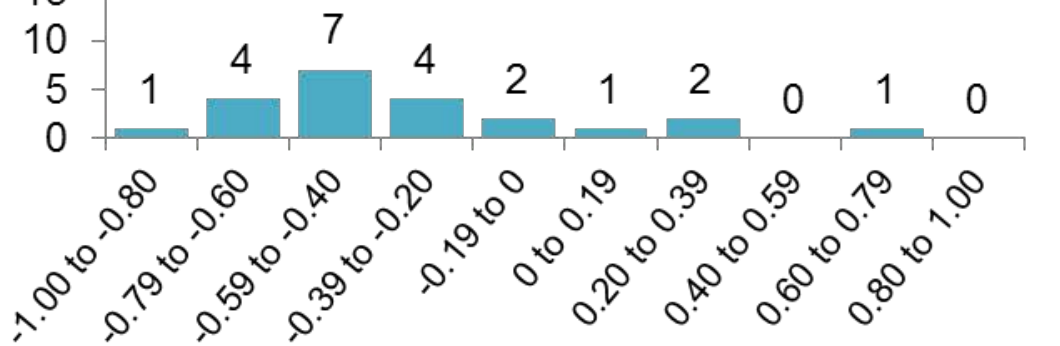
Correlation between

rearfoot EVE/INV and hip ADD/ABD

0

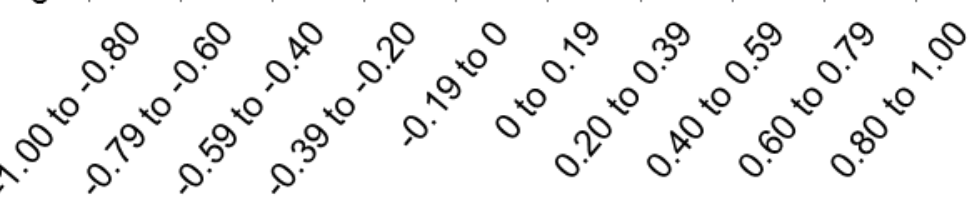

Correlation between

rearfoot ENV/INV and knee ADD/ABD

20
15
10
5
0

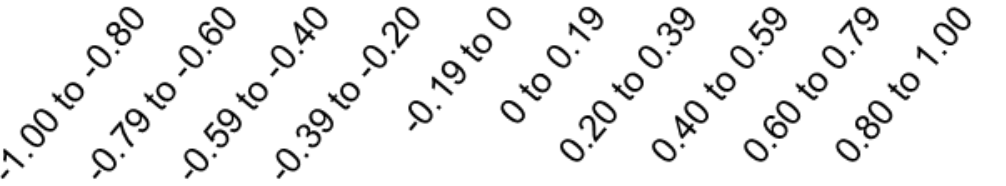

Correlation between

rearfoot $E R / I R$ and hip $A D D / A B D$

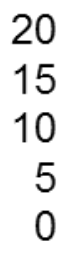

20
15
10
5

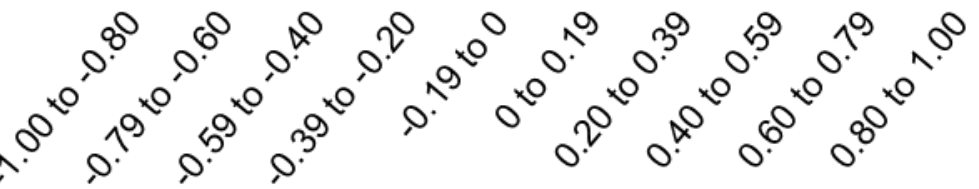

Correlation between

rearfoot $E R / I R$ and knee ADD/ABD

20
15
10
5
0

$\begin{array}{lllllllll}0 & 1 & 1 & 1 & 1 & 0 & 3 & 3\end{array}$

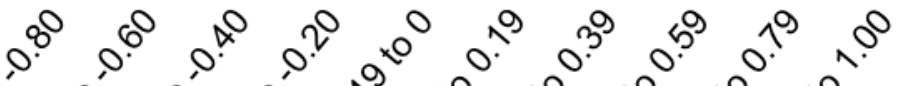

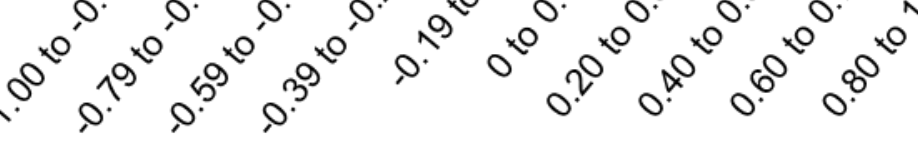

Correlation between rearfoot EVE/INV and hip IR/ER

\begin{tabular}{l|lllllllll}
5 & 0 & 1 & 0 & 0 & 0 & 1 & 1 & 1 & 1
\end{tabular}

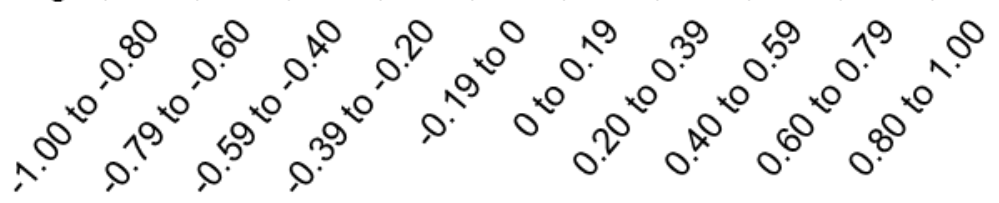

Correlation between rearfoot EVE/INV and knee IR/ER

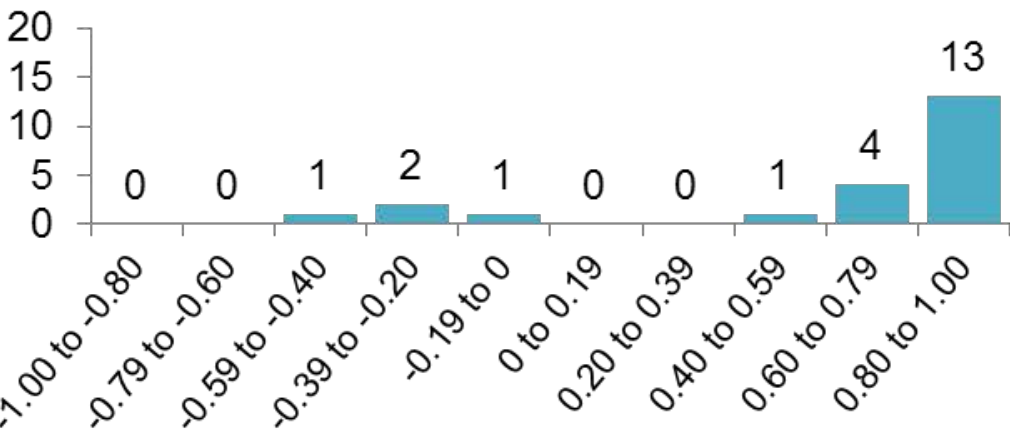

Correlation between rearfoot ER/IR and hip IR/ER

15

10

5

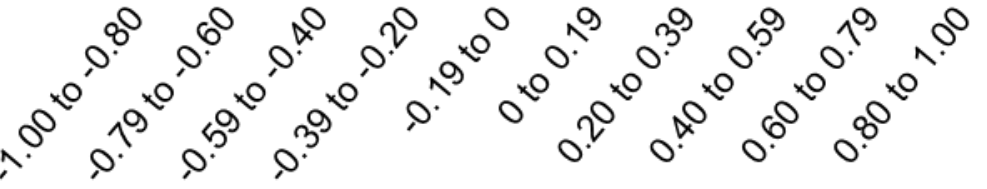

Correlation between rearfoot ER/IR and knee IR/ER 
Table 1. Presentation of the correlations and comparisons analyzed during walking and single-leg landing.

\begin{tabular}{lc}
\hline \multicolumn{1}{c}{ For rearfoot EVE/INV } & \multicolumn{1}{c}{ For rearfoot ER/IR } \\
\hline Correlations & Correlations \\
Rearfoot EVE/INV and hip ADD/ABD & Rearfoot ER/IR and hip ADD/ABD \\
Rearfoot EVE/INV and hip IR/ER & Rearfoot ER/IR and hip IR/ER \\
Rearfoot EVE/INV and knee ADD/ABD & Rearfoot ER/IR and knee ADD/ABD \\
Rearfoot EVE/INV and knee IR/ER & Rearfoot ER/IR and knee IR/ER \\
& \\
Comparisons of correlation coefficients & Comparisons of correlation coefficients \\
Rearfoot EVE/INV and hip ADD/ABD & Rearfoot ER/IR and hip ADD/ABD \\
vs. rearfoot EVE/INV and knee ADD/ABD & vs. rearfoot ER/IR and knee ADD/ABD \\
Rearfoot EVE/INV and hip ADD/ABD & Rearfoot ER/IR and hip ADD/ABD \\
vs. rearfoot EVE/INV and knee IR/ER & vs. rearfoot ER/IR and knee IR/ER \\
Rearfoot EVE/INV and hip IR/ER & Rearfoot ER/IR and hip IR/ER \\
vs. rearfoot EVE/INV and knee ADD/ABD & vs. rearfoot ER/IR and knee ADD/ABD \\
Rearfoot EVE/INV and hip IR/ER & Rearfoot ER/IR and hip IR/ER \\
vs. rearfoot EVE/INV and knee IR/ER & vs. rearfoot ER/IR and knee IR/ER \\
\hline
\end{tabular}


Table 2. Median (quartile deviation) of zero-lag cross-correlation coefficients between rearfoot motion and knee and hip joint motion during walking.

\begin{tabular}{lcc}
\hline & Rearfoot EVE/INV & Rearfoot ER/IR \\
\hline Hip ADD/ABD & $0.69(0.06)^{\mathrm{a}}$ & $0.84(0.08)^{\mathrm{b}}$ \\
Hip IR/ER & $0.06(0.22)$ & $-0.26(0.31)$ \\
Knee ADD/ABD & $0.37(0.13)$ & $0.51(0.20)^{\mathrm{c}}$ \\
Knee IR/ER & $-0.04(0.20)$ & $-0.41(0.21)$ \\
\hline
\end{tabular}

${ }^{\mathrm{a}}$ indicates a significantly stronger correlation than the correlations between rearfoot EVE/INV and knee ADD/ABD and between rearfoot EVE/INV and knee IR/ER during walking $(P<$ 0.001).

${ }^{\mathrm{b}}$ indicates a significantly stronger correlation than the correlations between rearfoot ER/IR and knee ADD/ABD and between rearfoot ER/IR and knee IR/ER during walking $(P<$ 0.001).

${ }^{\mathrm{c}}$ indicates a significantly stronger correlation than the correlation between rearfoot ER/IR and hip IR/ER $(P<0.001)$. 
Table 3. Mean coupling angles (SD) between rearfoot motion and knee and hip joint motion during walking.

\begin{tabular}{lcc}
\hline & Rearfoot EVE/INV & Rearfoot ER/IR \\
\hline Hip ADD/ABD & $41.7(7.2)^{\mathrm{a}}$ & $41.4(5.8)^{\mathrm{b}}$ \\
Hip IR/ER & $41.1(7.0)^{\mathrm{a}}$ & $40.8(5.9)^{\mathrm{b}}$ \\
Knee ADD/ABD & $63.9(5.1)$ & $63.8(4.9)$ \\
Knee IR/ER & $48.7(6.0)$ & $48.7(4.4)$ \\
\hline
\end{tabular}

${ }^{\mathrm{a}}$ indicates a significantly smaller than the coupling angles between rearfoot EVE/INV and knee ADD/ABD and IR/ER during walking $(P<0.001)$.

${ }^{\mathrm{b}}$ indicates a significantly smaller than the coupling angles between rearfoot ER/IR and knee ADD/ABD and IR/ER during walking $(P<0.001)$. 
Table 4. Median (quartile deviation) of zero-lag cross-correlation coefficients between rearfoot motion and knee and hip joint motion during single-leg landing.

\begin{tabular}{lcc}
\hline & Rearfoot EVE/INV & Rearfoot ER/IR \\
\hline Hip ADD/ABD & $0.70(0.13)$ & $0.92(0.05)^{\mathrm{a}}$ \\
Hip IR/ER & $0.89(0.07)$ & $0.92(0.07)$ \\
Knee ADD/ABD & $0.79(0.36)$ & $0.80(0.23)$ \\
Knee IR/ER & $0.87(0.13)$ & $0.79(0.21)$ \\
\hline
\end{tabular}

${ }^{a}$ indicates a significantly stronger correlation than the correlation between rearfoot ER/IR and knee IR/ER during single-leg landing $(P=0.001)$. 
Table 5. Mean coupling angles (SD) between rearfoot motion and knee and hip joint motion during single-leg landing.

\begin{tabular}{lcc}
\hline & Rearfoot EVE/INV & Rearfoot ER/IR \\
\hline Hip ADD/ABD & $36.8(8.9)^{\mathrm{a}}$ & $41.2(6.4)^{\mathrm{b}}$ \\
Hip IR/ER & $32.0(7.9)^{\mathrm{a}}$ & $36.8(6.9)^{\mathrm{b}}$ \\
Knee ADD/ABD & $48.3(9.1)$ & $53.4(9.3)$ \\
Knee IR/ER & $46.8(10.7)$ & $52.5(10.0)$ \\
\hline
\end{tabular}

${ }^{\mathrm{a}}$ indicates a significantly smaller than the coupling angles between rearfoot EVE/INV and knee ADD/ABD and IR/ER during single-leg landing $(P<0.001)$.

${ }^{\mathrm{b}}$ indicates a significantly smaller than the coupling angles between rearfoot ER/IR and knee ADD/ABD and IR/ER during single-leg landing $(P<0.001)$. 